\title{
Antecedentes preliminares del cultivo de bacalao de profundidad (Dissostichus eleginoides; Nototheniidae) en la región de Magallanes, Chile
}

\author{
Preliminary report on patagonian toothfish farming in \\ Magallanes region, Chile (Dissostichus eleginoides; Nototheniidae)
}

Pablo Gallardo Ojeda ${ }^{1}$

Los peces marinos de aguas frías se han convertido en una importante fuente alimenticia para el consumo humano, principalmente porque poseen altos contenidos de ácidos grasos de cadena larga, omega 3 y 6 , reconocidos como saludables para los seres humanos. Esto ha provocado una fuerte demanda y presión de pesca, que para el caso del bacalao de profundidad (Dissostichus eleginoides) ha sido enfatizada por su lento crecimiento y alto valor comercial. Bajo este escenario se vislumbran como posible alternativa de solución, el cultivo, aun cuando es sabido que el desarrollo de este tipo de tecnología es un proceso lento y lleno de desafíos (Reyes et al. 2012). Frente a este escenario, el Estado de Chile, ha desarrollado Programas Tecnológicos Estratégicos en Acuicultura y específicamente en bacalao de profundidad, como solución para la preservación del recurso, además de lograr la generación de nuevas o mayores divisas para el país. Bajo este contexto, la presente nota técnica tiene por objetivo entregar los primeros antecedentes referidos al cultivo de bacalao de profundidad en la región de Magallanes, comparando su tecnología de cultivo con el halibut del Atlántico (Hippoglossus hippoglossus), considerando similitudes biológicas entre ambas especies.

Las primeras experiencias de cultivo de esta especie han tenido como principal objetivo la captura y acondicionamiento de peces silvestres y cimentar las bases para futuras investigaciones del cultivo. Se ha demostrado, para el caso de peces marinos iteróparos, que es necesario contar con animales bien adaptados a la reproducción en cautiverio, para obtener progenies de buena calidad que sustenten una producción comercial y que al mismo tiempo permitan desvincularse de la obtención del medio natural. Los avances hasta ahora abarcan aspectos de captura y aclimatación de peces silvestres (Reyes et al. 2012), reproducción, en aspectos incipientes como desarrollo gonadal y tipo de ovas, nutrición (perfiles nutricionales, caracterización de microbiota intestinal), genética (diversidad, desarrollo de nuevos marcadores moleculares), salud (vigilancia de enfermedades de alto riesgo), ingeniería (desarrollo de estanques de reproductores, de incubación y cultivo larval) y de cultivo (tasas de crecimiento, supervivencia y alimentación).

\section{Captura y obtención de} peces para reproducción

La captura de bacalao de profundidad se realizó desde embarcaciones palangreras durante la faena regular de pesca en lugares, que incluyeron principalmente canales y fiordos magallánicos y Cabo de Hornos, a más de $1.500 \mathrm{~m}$ de profundidad, donde posteriormente fueron transportados hasta el Centro de Cultivos Marinos de la Universidad de Magallanes (Fig.

\footnotetext{
Centro de Cultivos Marinos Bahía Laredo. Departamento de Ciencias Agropecuarias y Acuícolas, Facultad de Ciencias, Universidad de Magallanes, Punta Arenas, Chile. $\sum$ pablo.gallardo@umag.cl
} 


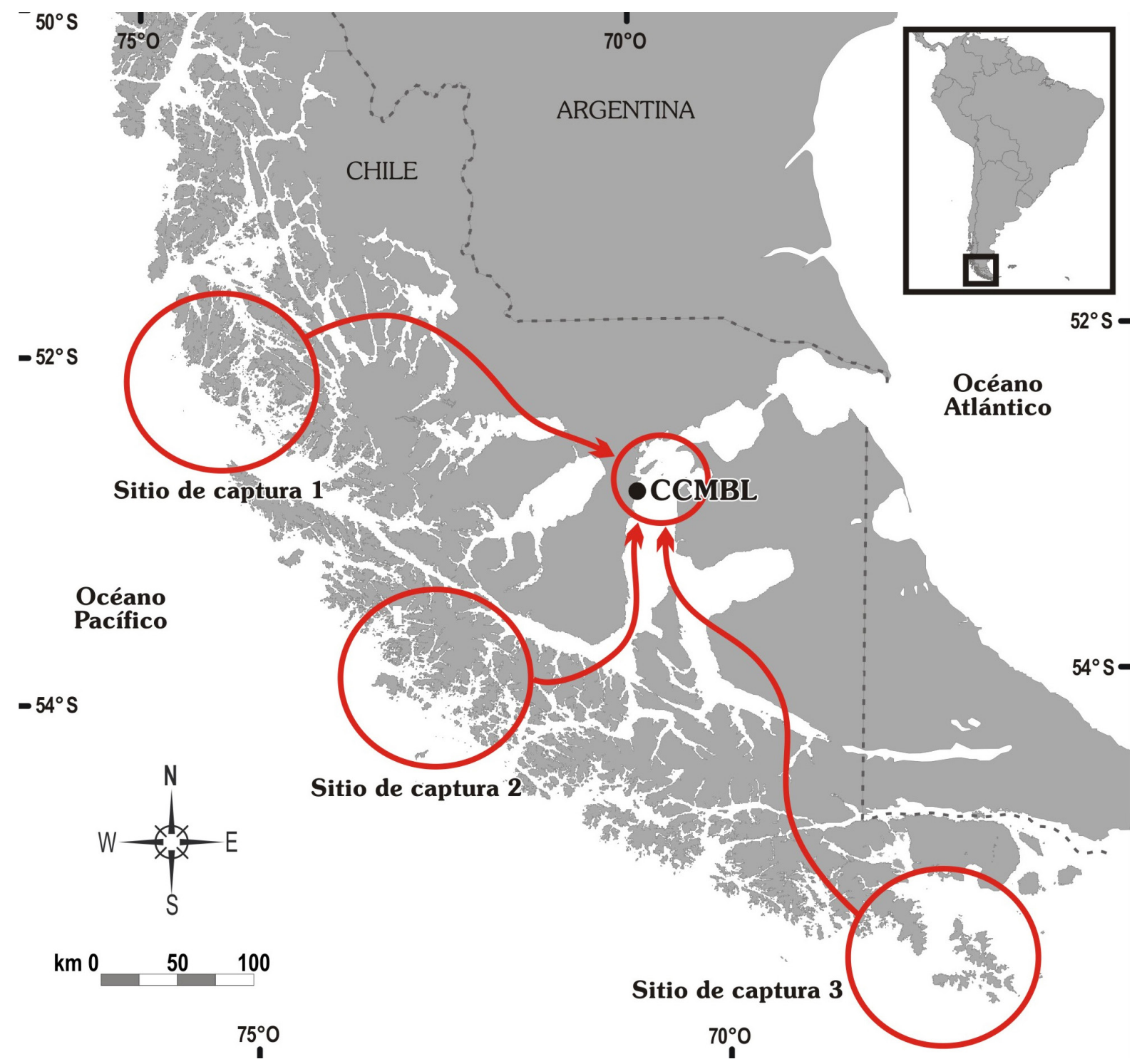

Fig. 1. Zonas de captura del bacalao de profundidad, siendo transportados hasta el Centro de Cultivos Marinos Bahía Laredo (CCMBL) de la Universidad de Magallanes.

1). La metodología de captura se realizó en base a lo descrito por Gallardo (2012a), la cual, resumidamente, consistió en seleccionar los peces desde el primer tercio de la cacea virada, a fin de obtener los que fueron izados con una menor velocidad de virado. Además, los peces seleccionados debían tener un tamaño entre 60 y $70 \mathrm{~cm}$ de longitud total y no presentar daño externo. Una vez retirado el anzuelo, los peces eran dispuestos en estanques de fibra de vidrio de 1.000 litros de capacidad con tapa, que contenían agua bombeada desde el mismo lugar de la captura y que además contaban con oxígeno comercial difundido mediante difusores de cerámica de porosidad igual a $500 \mu \mathrm{m}$, marca Pentair, modelo Point Four. La densidad de transporte máxima por estanque, fue de $10 \mathrm{~kg} / \mathrm{m}^{3}$. Luego de dispuestos los peces en los estanques, se monitoreó la temperatura, oxígeno y $\mathrm{pH}$, mediante un equipo digital marca Oxiguard, modelo Handy Polaris. La temperatura se mantuvo siempre bajo los $5^{\circ} \mathrm{C}, \mathrm{y}$ 
la concentración de oxígeno no fue inferior a 7 $\mathrm{mg} / \mathrm{L}$ o $70 \%$ de saturación, ni superior a los 12 $\mathrm{mg} / \mathrm{L}$ o $105 \%$ de saturación. Además, durante todo el traslado los peces se mantuvieron en completa oscuridad y sin alimentación.

La captura se realizó durante la época estival, ya que la reproducción de esta especie ocurre en invierno (Kock \& Kellermann, 1991) y por lo tanto se planificó capturar peces que estuviesen en período de reposo gonadal o de gametogénesis incipiente. La supervivencia promedio de peces entre 60 a $70 \mathrm{~cm}$ de longitud total, fue de un $20 \%$, lo cual es superior a los reportado por Reyes et al. (2012), que indican mortalidades cercanas al 90\%. Esto, quizás debido a que las capturas reportadas por dichos autores fueron realizadas a más de 2.000 kilómetros al norte de la zonas sindicadas como áreas de desove de la especie (Collins et al. 2010), por lo que, es posible que las condiciones ambientales, como por ejemplo la temperatura del agua, hayan causado un efecto adverso en la respuesta de los peces al confinamiento (Arana, 2009).

\section{Antecedentes de cultivo}

Inicialmente, el diseño del cultivo de bacalao de profundidad incluyó el análisis de otros cultivos de peces marinos de aguas frías,

Tabla 1. Características biológicas similares entre bacalao de profundidad y halibut del Atlántico.

\begin{tabular}{|c|c|c|c|}
\hline Característica & $\begin{array}{l}\text { Bacalao de } \\
\text { profundidad }\end{array}$ & Halibut del Atlántico & Referencia (*) \\
\hline Edad máxima & 40 a 50 años & 40 a 50 años & $\begin{array}{l}\text { Horn, (2002); Rønnestad, } \\
\text { (1988) }\end{array}$ \\
\hline $\begin{array}{l}\text { Longitudes máximas para } \\
\text { hembras }\end{array}$ & $226 \mathrm{~cm}$ & $300 \mathrm{~cm}$ & $\begin{array}{l}\text { Galleguillos et al. (2008); } \\
\text { Rønnestad, (1988) }\end{array}$ \\
\hline Distribución geográfica & aguas sub-antárticas & aguas sub-árticas & $\begin{array}{l}\text { Galleguillos et al. (2008); } \\
\text { Rønnestad, (1988) }\end{array}$ \\
\hline Batimetría & 70 a $2.500 \mathrm{~m}$ & 100 a $1.000 \mathrm{~m}$ & $\begin{array}{l}\text { North, (2002); Rønnestad, } \\
\text { (1988) }\end{array}$ \\
\hline $\begin{array}{l}\text { Alimentación juveniles y } \\
\text { reproductores }\end{array}$ & Peces óseos & Peces óseos & $\begin{array}{l}\text { Murillo et al. (2008); } \\
\text { Rønnestad, (1988) }\end{array}$ \\
\hline $\begin{array}{l}\text { Talla y edad de primera madurez } \\
\text { sexual en machos }\end{array}$ & $\begin{array}{l}70 \text { a } 90 \mathrm{~cm} \text {, entre } 7 \text { a } \\
11 \text { años }\end{array}$ & $\begin{array}{l}50 \text { a } 55 \mathrm{~cm} \text { de longitud total y } \\
4,5 \text { años. }\end{array}$ & $\begin{array}{l}\text { Arana, (2009); Jakupsstova \& } \\
\text { Haug, (1988). }\end{array}$ \\
\hline $\begin{array}{l}\text { Talla y edad de primera madurez } \\
\text { sexual en hembras }\end{array}$ & $\begin{array}{l}86 \text { a } 100 \mathrm{~cm}, \text { de } 9 \text { a } \\
12 \text { años }\end{array}$ & $\begin{array}{l}110 \text { a } 115 \mathrm{~cm} \text { de longitud y } \\
7 \text { años }\end{array}$ & $\begin{array}{l}\text { Sancho et al. (2003); } \\
\text { Jakupsstova \& Haug, (1988). }\end{array}$ \\
\hline Profundidad en la que desova & 800 a 1.200 & 1.000 metros & $\begin{array}{l}\text { Agnew et al. (1999); Hjort, } \\
\text { (1905) }\end{array}$ \\
\hline Tamaño y coloración ovas. & $\begin{array}{l}4,3 \text { a } 4,7 \mathrm{~mm} \mathrm{e} \\
\text { incoloros }\end{array}$ & 3 a $3,5 \mathrm{~mm}$ e incoloros & $\begin{array}{l}\text { Gallardo, (2012b), Rønnestad, } \\
\text { (1988) }\end{array}$ \\
\hline Tiempos de incubación & 90 días a $4^{\circ} \mathrm{C}$ & 13 días a $6^{\circ} \mathrm{C}$ & North, (2002); Gallardo, (2004) \\
\hline Talla de larva eclosionada & $\begin{array}{l}15 \mathrm{~mm} \text { de longitud } \\
\text { total }\end{array}$ & $7 \mathrm{~mm}$ de longitud total & $\begin{array}{l}\text { Hancet et al. (2008); Blaxter et } \\
\text { al. (1974) }\end{array}$ \\
\hline Alimentación larvas & Zooplancton & Zooplancton & $\begin{array}{l}\text { Galleguillos et al. (2008); } \\
\text { Rønnestad, (1988) }\end{array}$ \\
\hline Vejiga natatoria & Ausente & Ausente & $\begin{array}{l}\text { Oyarzún et al. (1988); } \\
\text { Rønnestad, (1988) }\end{array}$ \\
\hline
\end{tabular}

Fuente: elaboración propia. (*) Para cada característica indicada, el primer autor citado corresponde a bacalao de profundidad y el segundo a halibut del Atlántico. 
tales como Gadus morhua (Roy et al. 2007), Melanogrammus aeglefinus (Bromage \& Roberts, 1996), Anoplopoma fimbria (Guzmán et al. 2015) e Hippoglossus hippoglossus (Brown, 2010). Se seleccionó el cultivo de halibut del Atlántico (Hippoglossus hippoglossus), ya que se determinó que esta especie de pez plano presenta numerosas similitudes biológicas (Tabla 1), que indican la posibilidad de utilizar dicha tecnología de cultivo, como base para el desarrollo del cultivo de bacalao de profundidad. Además, el halibut del Atlántico había sido introducido a la región de Magallanes a finales de la década de los noventa y se ha completado todo el ciclo productivo en estanques en tierra (Gallardo, 2015).

Luego de capturados y trasladados, los peces se mantuvieron en estanques circulares de fibra de vidrio de 50.000 litros de volumen total, con flujo continuo de agua de mar filtrada a 500 $\mu \mathrm{m}$ y un caudal de 100 litros/min por 836 días, desde agosto de 2011 a diciembre de 2013. La temperatura del agua tuvo un promedio de 6,4 $\pm 1,4^{\circ} \mathrm{C}$ (Fig. 2). Los peces fueron alimentados a saciedad tres veces por semana, con una mezcla harinosa, rica en ácidos grasos altamente insaturados, además de aceite de pescado refinado, vitaminas e inmuno estimulante; todo esto en forma de pasta y entregado en formato de salchicha (Tabla 2). Su tasa de alimentación, expresada como porcentaje de su peso corporal fue en promedio de 0,6 $\pm 0,25 \%$ por día (Fig. 3). Para peces de un rango entre 1,9 a $11 \mathrm{~kg}$ y un periodo de 625 días de cautiverio, se obtuvo una tasa instantánea de crecimiento en peso promedio $0,06 \pm 0,08 \%$ por día, no existiendo diferencias significativas entre machos y hembras $(p>0,05)$ (Fig. 4). Considerando la información generada en confinamiento, se puede proyectar alcanzar los 6 kilogramos de peso promedio, luego de 48 meses en cautiverio, a partir de peces de $10 \mathrm{~g}$ de peso promedio inicial (Fig. 5), lo cual significaría acortar en un año el crecimiento natural (Arana, 2009).

\section{Antecedentes sanitarios, nutricionales y genéticos}

Además de la información de cultivo, se han estado desarrollando investigaciones en aspectos sanitarios, nutricionales y genéticos que son parte de publicaciones independientes, pero que incluyen protocolos para el fortalecimiento del sistema inmune, disminución del estrés post-captura; también se realizaron caracterizaciones anatómicas e histológicas de los peces, como base para el diagnóstico de patologías. Paralelamente, se realizó vigilancia epidemiológica, no detectando patógenos virales ni bacterianos en las muestras que se analizaron. Sólo se detectó presencia de cargas variables de parásitos metazoos, principalmente endoparásitos y escasamente ectoparásitos.

En cuanto a los antecedentes nutricionales, se desarrolló una línea base, abarcando los requerimientos nutricionales del pez y su alimento artificial y natural reportado previamente por Soto, (2015), con el objeto de obtener los perfiles completos de aminoácidos y ácidos grasos, tendientes a ajustar la dieta artificial a los requerimientos específicos de la especie.

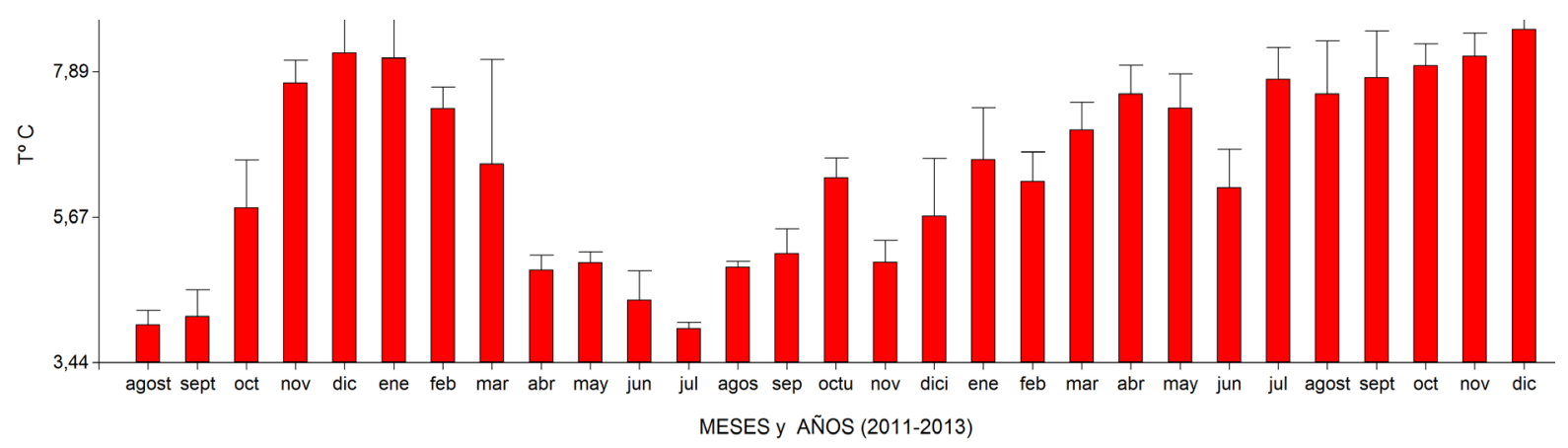

Fig. 2. Temperatura del agua promedio mensual para el estanque de cultivo de bacalao de profundidad (tiempo $=836$ días, $\mathrm{n}=3445, \min =3,5^{\circ} \mathrm{C}$, máx. $=10,4^{\circ} \mathrm{C}$ ). 


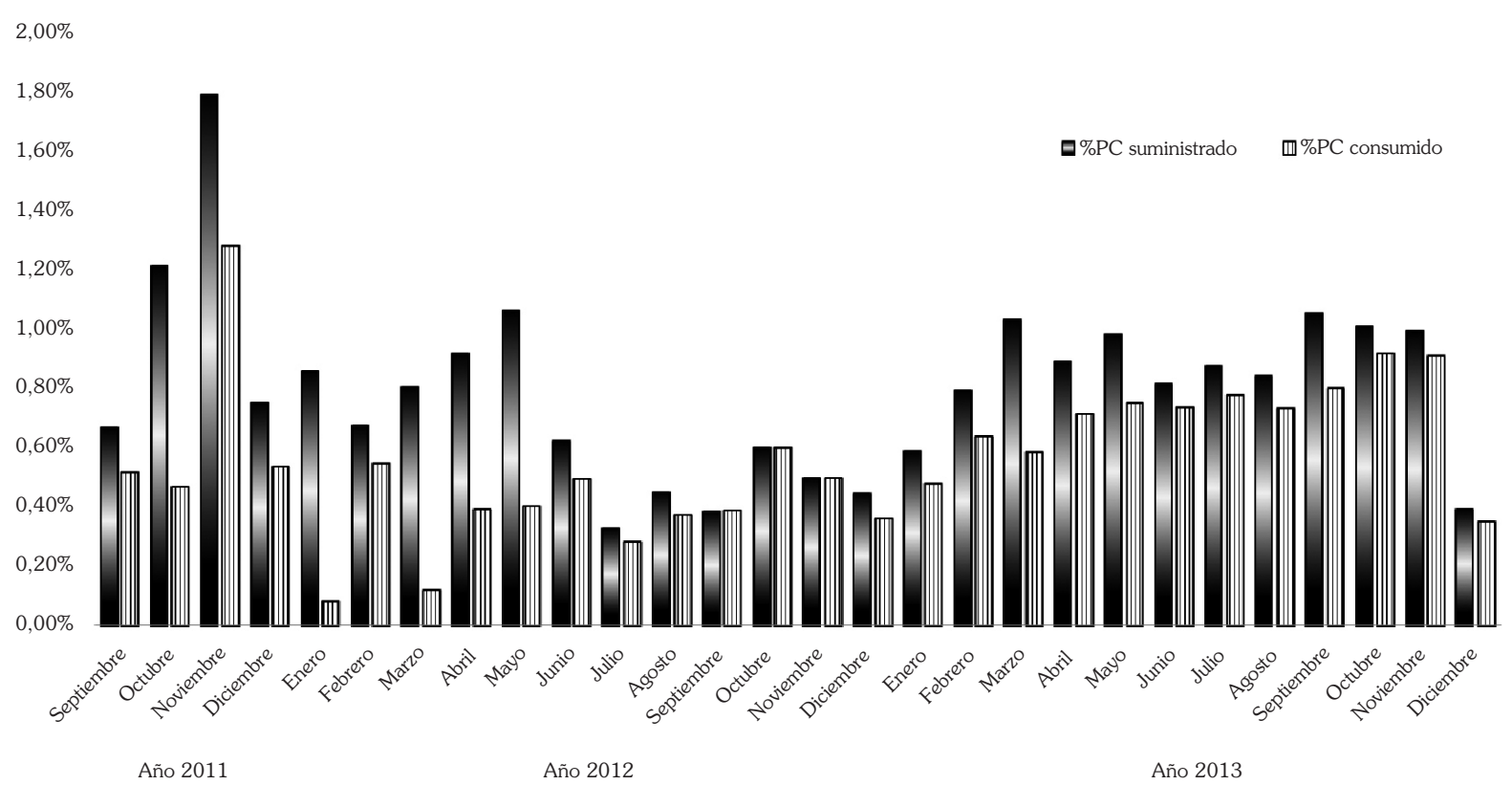

Fig. 3. Tasa de alimentación de bacalao de profundidad, expresada como porcentaje de su peso corporal suministrado como alimento (\%PC) (tiempo $=675$ días, $n=12$ peces).

En cuanto a los antecedentes genéticos, se extrajo ADN de muestras de peces de la pesquería industrial (Taggart et al. 1992), para luego cuantificar mediante Nanodrop y amplificación mediante PCR de cinco marcadores moleculares del tipo microsatélites (SSR) descritos para D. eleginoides por Reilly y Ward (1999), para los cuales, se diseñaron 4 pares de partidores (Primer-BLAST de NCBI). La heterocigosidad observada (Ho) ha sido alta para todos los loci analizados, con un rango desde 0,615 en el SSR cmrde2 hasta 0,844 en el SSR cmrde4. Los resultados obtenidos permiten caracterizar la diversidad genética de la población de $D$. eleginoides de la región de Magallanes (Araneda et al. en prensa). Paralelamente, se desarrolló una investigación asociada a la caracterización de la biota bacteriana gastrointestinal del bacalao de profundidad, utilizando metodologías cultivables y no cultivables. Esto permitió comparar las bacterias que se encontraban presente en peces silvestres con las de peces en proceso de aclimatación a condiciones de cultivo. Se encontraron diferencias significativas en cuanto a la biota, lo que confirma el efecto que provoca
Tabla 2. Ingredientes y análisis proximal del alimento entregado a bacalao de profundidad.

\begin{tabular}{lc}
\hline Ingredientes & Porcentaje \% \\
\hline Harina de Pescado & 45,28 \\
Aceite de pescado & 5,26 \\
Vitaminas e inmuno estimulantes & 0,15 \\
Envoltorio orgánico & 2,58 \\
Agua & 46,73 \\
& \\
\hline Ítem & Alimento (g/100 g) \\
\hline Proteínas & 31,8 \\
Lípidos & 6,5 \\
Cenizas & 7,1 \\
Extracto no nitrogenado & 5,8 \\
Humedad & 48,8 \\
Energía (Kcal/100 g) & 209 \\
\hline
\end{tabular}

la dieta artificial, sobre el tracto digestivo y sus implicancias nutricionales $e$ inmunitarias en el organismo de los peces, comparado con el ambiente natural (Urtubia et al. datos no publicados). 


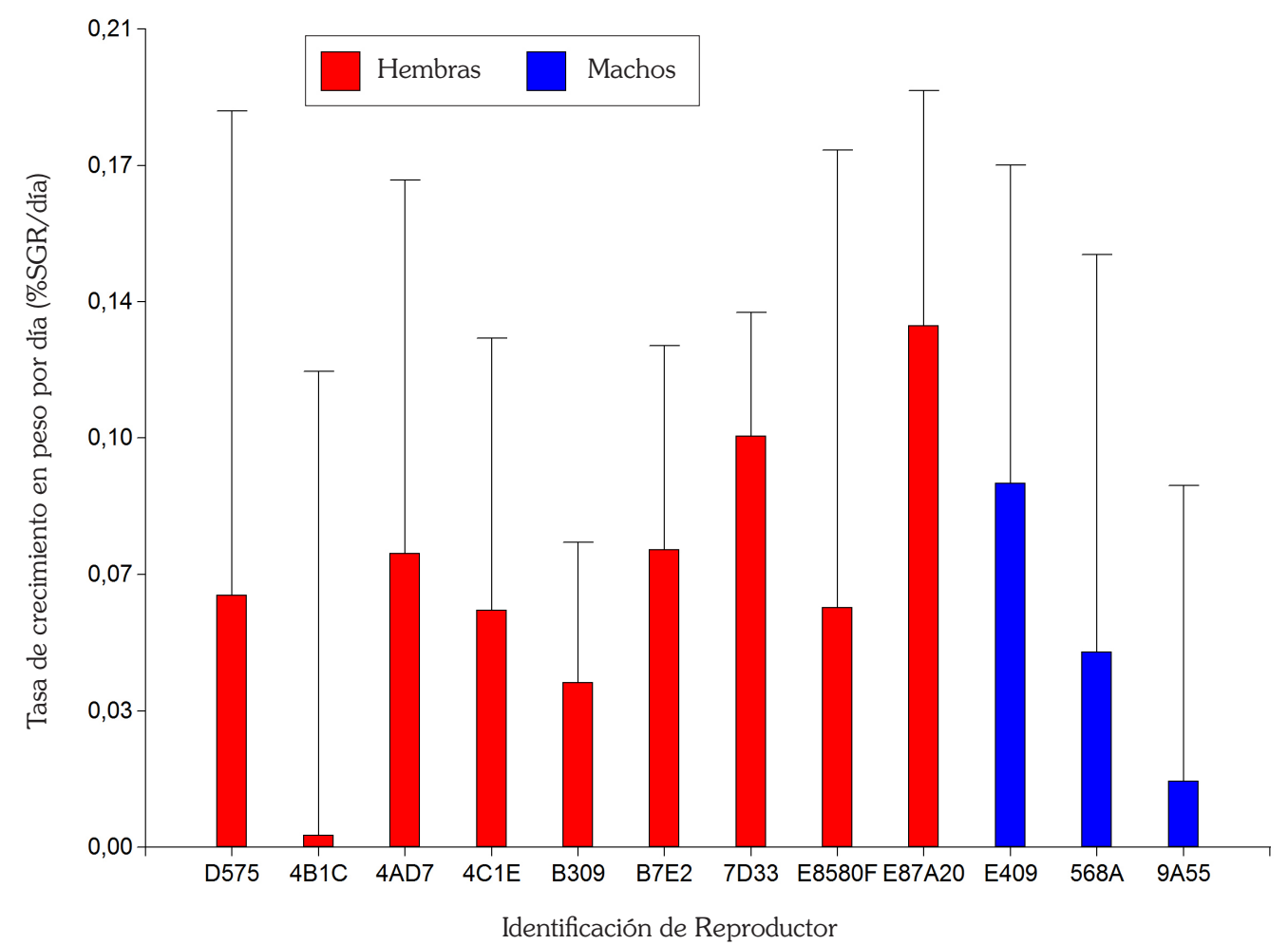

Fig. 4. Tasa instantánea de crecimiento en peso por día (SGR) de bacalao de profundidad $(\mathrm{n}=12)$, durante un período de 625 días de cautiverio. La numeración bajo cada barra en el eje X, es la identificación de cada pez.

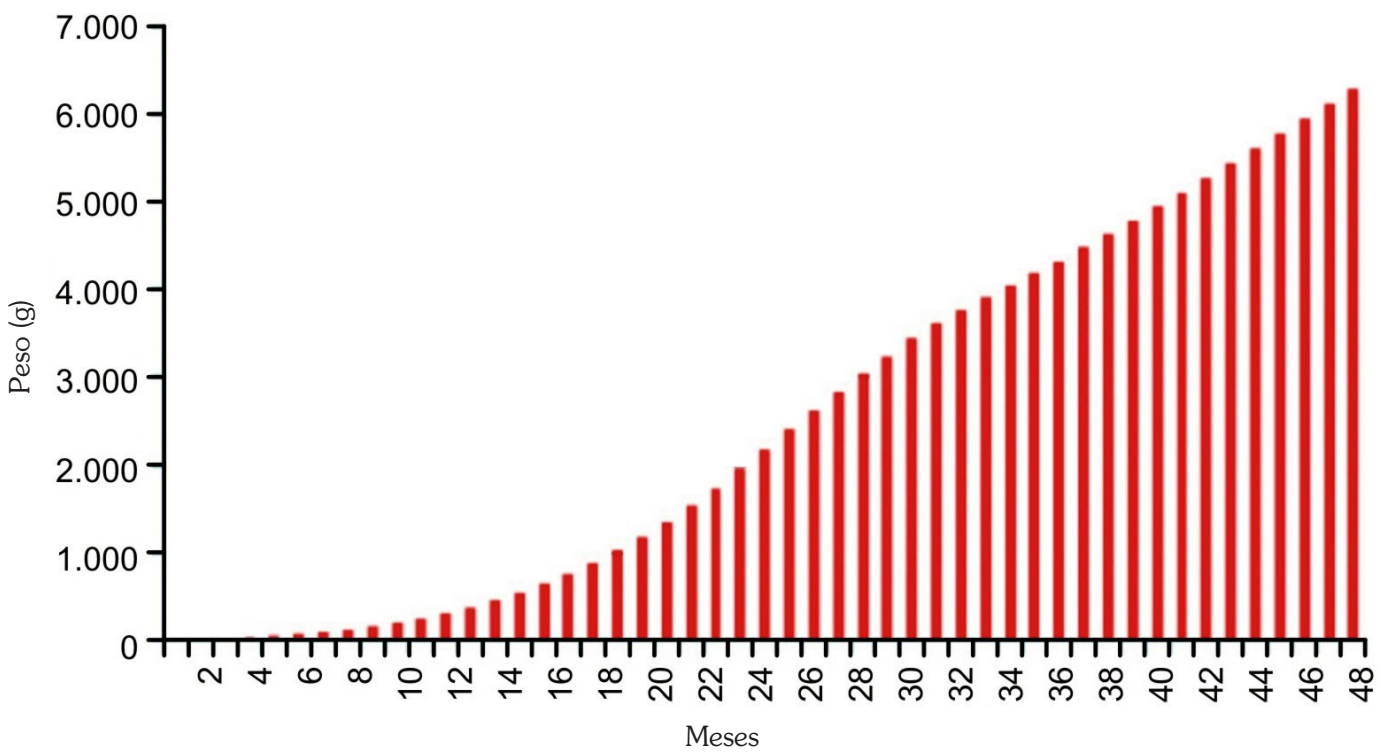

Fig. 5. Proyección de crecimiento en peso de bacalao de profundidad, hasta alcanzar los 6 kilogramos de peso promedio, en base a proyección de la tasa instantánea de crecimiento en peso (tiempo= 48 meses). 


\section{AGRADECIMIENTOS}

La presente investigación, se ha realizado con financiamiento FONDEF DA09i1002, más la colaboración de las empresas contrapartes e instituciones asociadas al proyecto, entre las que se destacan Globalpesca SpA, Instituto Antártico Chileno y la Facultad de Ciencias Agronómicas de la Universidad de Chile. Se agradece a los colegas de la Universidad de Magallanes su invitación a publicar este trabajo en una serie especial dedicada a esta especie; además, se agradecen los comentarios y sugerencias de dos revisores anónimos del manuscrito.

\section{LITERATURA CITADA}

Agnew, D. J., Heops, L., Jones C., Watson, A., Berkieta, K., \& Pearce, J. (1999). Depth distribution and spawning pattern of Dissostichus eleginoides at South Georgia. CCAMLR Science, 6, 19-36.

Arana, P. (2009). Reproductive aspects of the Patagonian toothfish (Dissostichus eleginoides) of Southern Chile. Latin American Journal of Aquatic Research, 37(3), 381-394.

Araneda, C., Lam N., Iturra P., Jilberto F., Cordoba V., \& Gallardo P. (en prensa). Genetic diversity and paternity exclusion for the Patagonian toothfish assessed with SSR markers". Latin American Journal of Aquatic Research.

Blaxter, J. H. S., Danielsen, D., Moksness, E., \& Øiestad, V. (1974). Description of the early development of halibut (Hippoglossus hippoglossus L.) and attemots to rear the larvae past first feeding. Marine Biology, 73, 99-107.

Collins, M, Brickle P, Brown J., \& Mark, B. (2010). The Patagonian Toothfish: Biology, Ecology and Fishery. Ecology and Fishery, 59, 227-300.

Gallardo, P. (2015). History and Methodology of Atlantic halibut (Hippoglossus hippoglossus) Farming in Southern, Chile. Aquaculture Conference 2015. Cutting Edge Science in Aquaculture. 23 26 August 2015. Le Corum, Montpellier,
France.

Gallardo, P. (2012a). Informe Técnico "Protocolo de Captura y Mantención a bordo de Embarcación". En Informe de Avance Científico - Tecnológico $\mathrm{N}^{\circ} 2$. Proyecto FONDEF DA09i1002.

Gallardo, P. (2012b). Informe Técnico "Obtención de Gametos de Bacalao de Profundidad Abordo". En Informe de Avance Científico - Tecnológico $\mathrm{N}^{\circ} 2$. Proyecto FONDEF DA09i1002.

Gallardo, P. (2004). Manual de cultivo del Halibut del Atlántico (Hippoglossus hippoglossus) en Magallanes. Proyecto FONDEF D99I1082 "Desarrollo de la Tecnología de cultivo de Halibut del Atlántico (Hippoglossus hippoglossus), en la XII región".

Galleguillos, R., Ferrada, S., Canales-Aguirre, C., Aedo, G., San Martin, M., \& Astete, S. (2008). Unidades poblacionales de bacalao de profundidad. Informe final FIP 2006-41.

Guzmán J., Luckenbach A., Goetz, F. W., Fairgrieve W. T., Middleton M. A., \& Swanson, P. (2015). Reproductive dysfunction in cultured sablefish (Anoplopoma fimbria). Bulletin of Fisheries Research Agency, 40, 111-119.

Hancet, S. M., Richard, G. J., Fenaughty, J. M., Dunn, A., \& Williams, M. J. H. (2008). A hypothetical life cycle for Antarctic toothfish (Dissostichus eleginoides) in the Ross Sea Region. CCAMLR Science, 15, 35-53.

Horn, P. L. (2002). Age and Growth of Patagonian toothfish (Dissostichus eleginoides) and Antartic toothfish (Dissostichus mawsoni) in waters from the New Zeland Subantartic to the Ross Sea, Antarctica. Fisheries Reserch, 56, 275-287.

Jakupsstovu, S. H., \& Haug, T. (1988). Growth, sexual maturation and spawning season of Atlantic halibut, Hippoglossus hippoglossus L. in Faroese waters. Fisheries Research, 6, 201-225.

Kock, W., \& Kellerman, H. (1991). Reproduction in Antarctic Notothenioids. A. Review. 
Antartic Science, 3(2), 125-150.

Murillo, C., Oyarzún, C., \& Fernández, I. (2008). Variación latitudinal y estacional en la dieta de Dissostichus eleginoides, Smitt, 1898 (Perciformes: Notothenidae) en ambientes profundos de la costa centro-sur de Chile. Gayana, 72(1), 94-101.

North, A. W. (2002). Larvas and juvenile distribution and growth of Patagonian toothfish around South Georgia. Antarctic Science, 14, 25-31.

Oyarzún, C., Campos, P., \& Valeria, H. (1988). Adaptaciones para la flotabilidad en Dissostichus eleginoides, Smitt 1898 (Pises, Perciforme, Notothenidae). Investigación Pesquera, 52(4), 455-466.

Reilly, A., \& Ward, R. D. (1999). Microsatellite loci to determine stock structure of the Patagonian toothfish Dissostichus eleginoides. Molecular Ecology, 8, 1753-1756.

Reyes, A., Kido, R., \& Moreno, C. A. (2012). Captura y mantención de Dissostichus eleginoides para conformar un plantel de reproductores. Latin American Journal of Aquatic Research, 40(4), 1066-1071.

Rønnestad, I. (1988). Rearing of Halibut (Hippoglossus hippoglossus). Fisheries Directorate, Oceanografic Research Institute, Aquaculture section. Aquaculture Satation at Austevoll.

Sancho, A., Ortíz-Von Halle, B., \& Naranjo, N. (2003). La pesca y el comercio del bacalao de profundidad, Dissostichus eleginoides en América del Sur: Una perspectiva regional. Informe de la Red Traffic.

Soto, I. (2015). Determinación e identificación del contenido estomacal de bacalao de profundidad silvestre (Dissostichus eleginoides, Smitt 1898), como apoyo para su desarrollo acuícola en la Región de Magallanes, Chile. Tesis de pregrado. Punta Arenas, Chile: Departamento de Ciencias y Recursos Naturales, Facultad de Ciencias, Universidad de Magallanes.

Taggart, J. B., Hynes, R. A., Prodöuhl, P. A., \& Ferguson, A. (1992). A simplified protocol for routine total DNA isolation from salmonid fishes. Journal of Fish Biology, 40, 963-965. 